\title{
Correlation between C-reactive protein levels and affecting factors for adiposity in apparently healthy Korean adolescents
}

\author{
Ki Eun Kim ${ }^{1 *}$, Yong-Jae Lee ${ }^{2}$, Yoon-Ho Shin ${ }^{2}$, Hye-Jung Shin ${ }^{3}$ \\ From 8th APPES Biennial Scientific Meeting \\ Darwin, Australia. 29 October - 1 November 2014
}

\begin{abstract}
Aims
Recent studies have shown that C-reactive protein is not just an indicator of cardiovascular disease (CVD) incidence and mortality. Thus, early detection of a continous increase in CRP concentrations may be useful in predicting subsequent development of CVD or metabolic syndrome.

The objective of this study was to analyze high sensitivity C-reactive protein (hs-CRP) in apparently healthy young Korean adolescents and determine confounding factors for high hs-CRP in this population.
\end{abstract}

\section{Methods}

We enrolled 197 middle school students (93 boys and 104 girls) who participated in a general health check-up at a tertiary hospital in Seoul. We measured height, weight, waist circumference and blood pressure and investigated hs-CRP concentrations, insulin levels, insulin resistance and lipid profiles. Hs-CRP levels were measured using the Behring BN II nephelometer (Dade Boering, Marburg, Germany) and log-transformed for analysis.

\section{Results}

hs-CRP concentration was significantly higher in boys than in girls $(\mathrm{P}=0.012)$. Pearson's correlation coefficients revealed a significant correlation between log- transformed hs-CRP and BMI $(\mathrm{r}=0.24, \mathrm{P}=0.0008)$, waist circumference $(\mathrm{r}=0.24, \mathrm{P}=0.0007)$, systolic $(\mathrm{r}=0.17, \mathrm{P}=0.019)$ and diastolic blood pressure $(\mathrm{r}=0.23, \mathrm{P}=0.014)$, and ALT $(\mathrm{r}=0.16, \mathrm{P}=0.023)$. In stepwise multivariate linear regression analysis, sex (male gender), waist circumference, and diastolic blood pressure were positively and fasting serum HDL-cholesterol level was negatively associated with log-transformed hs-CRP.

\section{Conclusions}

We found that there exists a gender difference in hsCRP concentrations in apparently healthy adolescents and that log-transformed hs-CRP concentrations were positively associated with male, waist circumference and diastolic blood pressure and negatively associated with HDL-cholesterol level. The gender difference and the contibuting factors for hs-CRP found in these healthy adolescents suggests a possibly relevant pathophysiological mechanism involved in the increase of cardiovascular risk associated with childhood obesity.

\section{Authors' details}

'Department of Pediatris, CHA Gangnam Medical center, CHA University, Seoul, Korea. ${ }^{2}$ Department of Family Medicine, Yongin Severance hospital, Yensei University, Geonggi, Korea. ${ }^{3}$ Department of Pediatrics, National Medical Center, Seoul, Korea.

Published: 28 April 2015

\section{doi:10.1186/1687-9856-2015-S1-P69}

Cite this article as: Kim et al:: Correlation between C-reactive protein levels and affecting factors for adiposity in apparently healthy Korean adolescents. International Journal of Pediatric Endocrinology 20152015 (Suppl 1):P69. 